\title{
Erratum: Ultrafine Entanglement Witnessing [Phys. Rev. Lett. 118, 110502 (2017)]
}

\author{
Farid Shahandeh, Martin Ringbauer, Juan C. Loredo, and Timothy C. Ralph \\ (Received 14 November 2017; published 28 December 2017)
}

DOI: 10.1103/PhysRevLett.119.269901

The original proof of Theorem 1 within the Supplemental Material of our Letter relied on Lemma 3, stating that any separable mixed quantum state $\hat{\sigma} \in \mathcal{S}_{\text {sep }}$, which satisfies $\operatorname{Tr} \hat{C} \hat{\sigma}=c$ can be decomposed as $\hat{\sigma}=p|a, b\rangle\langle a, b|+(1-p) \hat{\sigma}_{\text {re }}$ for some $p>0$ such that $|a, b\rangle\langle a, b|$ is a pure product state satisfying $\langle a, b|\hat{C}| a, b\rangle=c$ where the state $\hat{\sigma}_{\text {re }}$ is the remainder. However, decomposing $\hat{\sigma}$ into pure states requires those states to be within the range of $\hat{\sigma}$, a necessary condition which is not guaranteed by the previous proof. As a consequence, it was brought to our attention that it is possible to construct counterexamples to Theorem 1 in which the optimal state $\hat{\sigma}_{\mathrm{opt} ; X} \in \mathcal{S}_{\mathrm{sep} ; X}$ to the test operator $\hat{L}$ at a given constraint value $c$ is a mixed state of rank two. For instance, considering the constraint and test operators $\hat{C}=|00\rangle\langle 00|-| 11\rangle\langle 11|$ and $\hat{L}=|00\rangle\langle 00|+| 11\rangle\langle 11|$, for any given value of $c$, the optimal separable state is given by $\hat{\sigma}_{\text {opt } ; c}=(1+c) / 2|00\rangle\langle 00|+$ $(1-c) / 2|11\rangle\langle 11|$. This state has a rank of two except at $c=-1$ and $c=1$.

A slightly modified version of Theorem 1 can be stated and proved as per below.

Correction to Theorem 1. For a given constraint value $c$, the optimal state $\hat{\sigma}_{\mathrm{opt} ; X} \in \mathcal{S}_{\mathrm{sep} ; X}$ to the test operator $\hat{L}$ is at most of rank two with $\operatorname{Tr} \hat{C} \hat{\sigma}_{\mathrm{opt} ; X}=c$.

Proof.-Suppose that the optimal state is mixed and has the form $\hat{\sigma}_{\mathrm{opt}, c}=\sum_{i} p_{i}\left|a_{i}, b_{i}\right\rangle\left\langle a_{i}, b_{i}\right|$, where each $\left|a_{i}, b_{i}\right\rangle\left\langle a_{i}, b_{i}\right|$ is a pure product state and $\sum_{i} p_{i}=1$. From Eq. (2.3) in the Supplemental Material of our Letter, it follows that

$$
g_{c}=\operatorname{Tr} \hat{L} \hat{\sigma}_{\mathrm{opt} ; c}=\sum_{i} p_{i} \operatorname{Tr} \hat{L} \hat{\sigma}_{\mathrm{opt} ; c_{i}}=\sum_{i} p_{i} g_{c_{i}}
$$

where $\hat{\sigma}_{\text {opt } ; c_{i}}=\left|a_{i}, b_{i}\right\rangle\left\langle a_{i}, b_{i}\right|$ must be the optimal separable state over $\mathcal{S}_{\text {sep } ; c_{i}}$ with the optimal value $g_{c_{i}}=\operatorname{Tr} \hat{L} \hat{\sigma}_{\text {opt } c_{i}}$. As we have assumed that the optimal point $\hat{\sigma}_{\text {opt } ;}$ is a mixed state, we conclude that $c_{i} \neq c$ for all $i$. We now assume that the indices are ordered such that, for some $k, c_{1} \leq \cdots \leq c_{k}<c<c_{k+1} \leq \cdots$. Consequently, the inclusion of sets $\mathcal{S}_{\text {sep; } c_{1}} \subseteq \cdots \subseteq \mathcal{S}_{\text {sep; } c_{k}} \subset$ $\mathcal{S}_{\text {sep;c }} \subset \mathcal{S}_{\text {sep } ; c_{k+1}} \subseteq \cdots \subseteq \mathcal{S}_{\text {sep }}$, implies that $g_{c_{1}} \leq \cdots \leq g_{c_{k}} \leq g_{c} \leq g_{c_{k+1}} \leq \cdots \leq g_{\mathrm{s}}$. Now consider the convex polytope defined by the convex hull of the elements of this decomposition in the Cartesian coordinates $(c, \ell)$, where $c=\operatorname{Tr} \hat{C} \hat{\varrho}$ and $\ell=\operatorname{Tr} \hat{L} \hat{\varrho}$ for any state $\hat{\varrho}$. It is well known that for any given $c$ the maximum (and minimum) of $\ell$ over this polytope must occur on its boundaries. Hence, the optimal point can always be described as a convex combination of two vertices. In other words, there must exist an optimal state $\hat{\tau}_{\mathrm{opt} ; c}$ of rank two, so that $\operatorname{Tr} \hat{L} \hat{\tau}_{\mathrm{opt} ; c}=\operatorname{Tr} \hat{L} \hat{\sigma}_{\mathrm{opt} ; c}=g_{c}$.

An important consequence of the above argument is that the full separability curve can indeed be obtained by optimizing only over pure states and taking the convex hull of the resulting curve in $(c, \ell)$ coordinates, rendering an actual optimization over rank two mixed states unnecessary. This follows immediately from the fact that for some values of $c$, the optimal states are pure (e.g. the pure states in the decomposition of $\hat{\sigma}_{\mathrm{opt} ; c}$ ), and that the optimal state for any value of $c$ is at most a convex combination of two other states on the curve. Furthermore, this proof can easily be extended to show that the optimal point for UEW with $n$ constraints, can always be written as a separable state of rank at most $n+1$.

We also rephrase the statement of Theorem 2 in a more precise form to avoid possible ambiguities.

Correction to Theorem 2. The necessary condition for the separable, positive operators $\hat{C}$ and $\hat{L}$ to detect entanglement via UEW is that $\hat{C}$ and $\hat{L}$ are not diagonal in a common product basis.

In light of the above analysis, corrections, and clarifications we conclude that the separability curve and the experimental analysis presented in our Letter are correct and no modifications are required. Furthermore, while these considerations change the details of Theorem 1, they do not change the main result of our Letter, nor its implications for entanglement witnessing.

We thank M. Gachechiladze, O. Gühne, and N. Wyderka for bringing to our attention the counterexample to Theorem 1 reproduced above, and for pointing out subtleties regarding Theorem 2. 